WIENER SLAVISTISCHES JAHRBUCH, Band 56/2010, 215-234

(C) 2010 by Österreichische Akademie der Wissenschaften, Wien

STEFAN SIMONEK

NATALIA TSIKRA

\title{
Die Rezeption Émile Zolas in Lemberger Periodika um 1900
}

Auf den folgenden Seiten soll im Rahmen einer weiter angelegten Fragestellung nach der Aufnahme Émile Zolas in den mitteleuropäischen Literaturen ${ }^{1}$ (mit einem Schwerpunkt auf der Wende vom 19. hin zum 20. Jahrhundert) der ganz vorrangig bibliographisch-quellenkundlich ausgerichtete Versuch unternommen werden, die Berichterstattung zu Zola in ausgewählten Lemberger Periodika, konkret in der polnischen Tageszeitung „Kurjer Lwowski“ [Lemberger Kurier], in der ukrainischen Tageszeitung „Dilo“ [Die Tat] sowie in der Lemberger ukrainischen Monatsschrift „Literaturno-naukovyj visnyk“ [Literarisch-wissenschaftlicher Bote] nachzuzeichnen. ${ }^{2}$ Die Entscheidung für gerade diese drei Periodika erklärt sich dabei einmal aus der Möglichkeit heraus, über das Nebeneinanderstellen unterschiedlicher medialer Formate wie Tageszeitung und Monatsschrift auch unterschiedliche, teilweise synchron verlaufende Rezeptionswege aufzuzeigen; daneben soll die Kombination von polnischen und ukrainischen Blättern aber auch die multilinguale, von Konkurrenz wie Konvergenz gleichermaßen geprägte kulturelle Konstellation in der Hauptstadt des Kronlandes Galizien und Lodomerien am Ostrand der Donaumonarchie entsprechend zum Ausdruck bringen ${ }^{3}$ - das, was von polnischer Seite her an Zolas Biogra-

${ }^{1}$ Grundlage des vorliegenden Beitrags waren zwei Referate im Rahmen der international besetzten Tagung „Die Rezeption Émile Zolas in Mitteleuropa / La réception d'Émile Zola en Europe centrale" (Wien, 3.-5. Dezember 2009), die von Norbert Bachleitner (Wien) in Zusammenarbeit mit Tone Smolej (Ljubljana) organisiert wurde.

2 Der Abschnitt über die beiden Tageszeitungen „Kurjer Lwowski“ und „Dilo“ wurde von Natalia Tsikra, jener über die Monatsschrift „Literaturno-naukovyj visnyk“ von Stefan Simonek verfasst.

3 Zur polnischen und ukrainischen (ruthenischen) Presse der Donaumonarchie vgl. Harald Binder, Die polnische Presse, in: Die Habsburgermonarchie 1848-1918. Hrsg. v. Helmut Rumpler u. Peter Urbanitsch, Bd. VIII/2, Wien: Verlag der Österreichischen Akademie 
phie wie auch an seinen Werken rezipiert wurde, musste aus der zuvor erwähnten konkurrierenden Perspektive ja nicht automatisch auch für die ukrainische Seite von Relevanz sein (auch wenn sich in Bezug auf die Berichterstattung zu Zola durchaus signifikante Parallelen zwischen den einzelnen Blättern ergeben, die nicht zuletzt auch auf den Umstand zurückzuführen sind, dass einzelne wesentliche Proponenten der galizischen Zola-Rezeption wie allen voran Ivan Franko gleich für mehrere Lemberger Blätter tätig waren).

Innerhalb der kulturellen Konstellation in der galizischen Hauptstadt spielten naturgemäß die polnische und die ukrainische Kultur und Literatur eine dominierende Rolle, weiters war (als kulturelle Formation der Reichshauptstadt) aber auch die Wiener Moderne von Interesse und daneben auch die englische und die französische Literatur. Letztere gehörte etwa über Symbolismus (vor allem Baudelaire und Verlaine, daneben aber auch die Belgier Rodenbach und Maeterlinck) und Naturalismus $\mathrm{zu}$ den führenden und einflussreichsten westeuropäischen Literaturen im Ausgang des 19. Jahrhunderts, die auch in den slawischen Literaturen ihre entsprechende Resonanz gefunden hat. Deshalb zogen gerade die französischen Schriftsteller die Aufmerksamkeit von Übersetzern aus den verschiedensten europäischen Literaturen auf sich, und die literarischen Zeitschriften beeilten sich, möglichst früh derartige Übersetzungen zu publizieren. Am Ende des 19. Jahrhunderts - und damit innerhalb des hier abgesteckten Berichtszeitraums - interessierten sich sowohl die literarischen als auch die politischen Zeitschriften in Lemberg für Zola als Repräsentant des Naturalismus.

In Galizien, insbesondere in Lemberg, erschienen fast zur gleichen Zeit zwei der auflagenstärksten politischen Tageszeitungen, „Dilo“ (ab 1880) in ukrainischer Sprache (der erste Redakteur war Volodymyr Barvins'kyj) und „Kurjer Lwowski“ (ab 1883) in polnischer Sprache (der erste Redakteur war Bolesław Wisłouch). Diese zwei Zeitungen Galiziens waren mit der nationalen Bewegung „Narodnyj Ruch“ verbunden und kämpften für die Rechte und Freiheiten der ukrainischen und der polnischen Volksgruppe innerhalb der Donaumonarchie. Texte von Émile Zola wurden zuerst auf den Seiten des „Kurjer Lwowski“ veröffentlicht. Am 5. Juni 1886 wurde die Übersetzung einer Passage aus den Contes à Ninon unter dem Titel $W y$ chowanie dzieci [Die Kindererziehung] in Nr. 155 dieser Zeitung gedruckt. Die Erzählung erschien unter der Rubrik „Z nowych opowiadań Emila Zoli“ [Aus den neuen Erzählungen von Émile Zola].

der Wissenschaften 2006, S. 2037-2090; ders.: Die ruthenische Presse, in: ebd., S. 20912126; zu „Dilo“vgl. weiters Jurij Hryhorovyč Šapoval, Dilo (1880-1939 rr.). Postup ukrajins'koji suspil'noji dumky, L'viv: Nacional'na akademija nauk Ukrajiny 1999; zu „Di10 “ und „Literaturno-naukovyj visnyk" vgl. weiterhin die entsprechenden Einträge von I. Kedryn-Rudnytsky bzw. V. Doroshenko in: Encyklopedia of Ukraine, vol. I, Toronto Buffalo - London: University of Toronto Press 1984, S. 671 bzw. vol. III, 1993, S. 157 f. 
Sechs Tage später wurde in Nr. 161 des „Kurjer Lwowski“ unter der Rubrik „Aus der ausländischen Literatur“ eine Anzeige zum neuen Roman Zolas L'œuvre veröffentlicht. Der Verfasser, der sich hinter dem Pseudonym Admir verbirgt, beginnt seinen Artikel mit folgenden Worten: ,[...] zajmują świat literacki dwa romanse nowe największych pisarzy współczesnych, Zoli L'œuvre [...]" (2). ${ }^{4}$ [Die literarische Welt ist von zwei neuen Romanen großer zeitgenössischer Autoren entzückt, nämlich von Zolas L'euvre.] Dann bemerkt er, dass dieser Roman vom geheimnisvollen Leben eines Künstlers handle, und gibt den Inhalt kurz wieder. Die Helden des Romans nennt er Apostel des Naturalismus. Seinen Artikel über den neuesten Roman von Émile Zola beendet der Kritiker mit folgenden Worten: „Cokolwiek o nim powie krytyka, najzagorzalsi przeciwnicy jego nawet przyznać musza, że wielki pisarz właśnie sceny drastyczne po raz może pierwszy traktował w L'œuvre z nadzwyczajną dyskrecją“ (3). [Was auch immer die Kritik über ihn sagen mag, auch seine schärfsten Kritiker müssen anerkennen, dass der große Schriftsteller gerade die drastischen Szenen in L'œuvre vielleicht zum ersten Mal mit ungewöhnlicher Diskretion behandelt hat.]

Im Januar 1887 erschien in Nr. 18 des „Kurjer Lwowski“ eine Anzeige unter der Rubrik „Teatr, literatura, sztuka“ [Theater, Literatur, Kunst], aus der hervorgeht, dass Zolas neuer Roman La terre bald auf den Regalen der Buchläden greifbar sein werde. Dort findet sich auch eine kurze Inhaltsangabe des Romans, und in der Anzeige wird auch eine literarische Analyse des Werks geboten. Gegenstand der Intrige ist laut dieser Darstellung die Teilung von Ländereien zwischen zwei Brüdern, und das Thema der Romanhandlung ist das Schicksal des Bauern. Der Verfasser dieser Anzeige (er ist leider unbekannt) macht darauf aufmerksam, dass die Ereignisse der Romanhandlung im Winter beginnen und enden. Dies sei als symbolische Darstellung des Bauernschicksals, das hart wie Eis ist, zu verstehen. Der Kritiker erörtert daneben auch die literarischen Pläne von Zola, ohne freilich einen Hinweis auf die Quelle seiner Informationen zu geben. Er schreibt, dass der französische Schriftsteller außer diesem Roman noch fünf weitere Werke zu schreiben beabsichtige, die folgenden Themen gewidmet sein sollen: der Eisenbahn, der Börse, dem Journalismus und dem Krieg. Aber er deutet auch darauf hin, dass dies dann seine letzten Prosaarbeiten sein sollten, denn der Meinung des Verfassers nach sollte sich Zola danach dem Drama widmen.

Der „Kurjer Lwowski“ führte auch die Tradition ein, über viele Nummern hinweg polnische Übersetzungen von Erzählungen und Romanen zeitgenössischer europäischer, amerikanischer und heimischer Autoren zu veröffentlichen; der Leser konnte diese Fortsetzungen dann selbst zu einem Buch zusammenbinden. Unter den auf diese Weise präsentierten Autoren finden sich u. a. auch französische Schriftstel-

${ }^{4}$ Die entsprechende Seitenzahl wird hier und im Folgenden in runder Klammer direkt hinter dem betreffenden Zitat im Haupttext vermerkt. 
ler, wie Guy de Maupassant, Albert Delpit, Emmanuel Arène und natürlich auch Émile Zola, dessen Roman Jacques Damour vom 16. bis zum 27. Februar 1887 in Fortsetzungen im „Kurjer Lwowski“ veröffentlicht wurde.

Durch die immer größere Popularität von Werken der polnischen und der ausländischen Literaturen wurde die Redaktion des „Kurjer Lwowski“ zur Erweiterung gezwungen. Als Folge daraus entstand ein eigenes wissenschaftlich-literarisches Heft mit dem Titel „Tydzień“ [Die Woche]. In der „Woche“ wurden ganz vorrangig Werke von polnischen, ukrainischen, tschechischen und bulgarischen Autoren veröffentlicht, Zola dagegen blieb einer der wenigen westeuropäischen Schriftsteller, von dem eigene Werke und (insgesamt drei) Beiträge über ihn selbst publiziert wurden.

In Nr. 32 der „Woche“ wurde 1895 auch der Artikel Zola o swej nowej powieści [Zola über seinen neuen Roman] veröffentlicht; es handelt sich dabei um eine Übersetzung von Zolas Bericht über den Entstehungsprozess seiner Trilogie Lourdes Rome - Paris für die Pariser Zeitschrift „Le Matin“, in der Zola über Interna des schöpferischen Prozesses referiert. Besonderes Interesse gilt dabei der Tatsache, dass Zola zwei Kisten voll von Dokumenten und sechshundert Seiten Notizen aus Rom mitgebracht und allein zum Ordnen dieser Notizen sechs Monate Zeit gebraucht hat. Dieser Bericht endet mit den Eindrücken Zolas von Rom und vom Vatikan.

1896 wurde in Nr. 26 der „Woche“ ein Artikel mit dem Titel Zola veröffentlicht. Dies war die Übersetzung eines Artikels aus „Le Figaro“, nämlich Zolas Reaktion auf die Plagiatsbeschuldigung seitens eines Journalisten, nachdem sein Roman Rome erschienen war. Bemerkenswert sind hier die Beweise, die Zola zu seinem eigenen Schutz aufbringt: Der Autor führt nämlich an, dass er in den dreißig Jahren seines schöpferischen Lebens über ein Tausend Menschengestalten zum Leben erweckt habe und diese seine „Kinder“ seien. Daher habe er es auch nicht nötig, „fremde Kinder" zu stehlen.

In Nr. 40 der „Woche“ aus dem Jahr 1902 wurde in der Rubrik „Życiorysy, wspomnienia, listy" [Lebensbilder, Erinnerungen, Briefe] ein umfangreicher und interessanter, drei Seiten langer Artikel mit dem Titel Émile Zola veröffentlicht. In diesem Artikel, der lediglich mit „St. W.“ unterzeichnet ist, erörtert der Verfasser sein Verständnis des Naturalismus bei dem französischen Schriftsteller und polemisiert mit dessen Kritikern. Dieser Artikel soll genauer besprochen werden, er beginnt sehr poetisch:

Literatura $\mathrm{z}$ „przedwczoraj“, jak ją nazywają, to znaczy naturalizm lat 80 -ych, miał na swoim horyzoncie dwa słońca. Od północy świecił nam Henryk Ibsen, zalewając białym zimnym światłem skały i pomarzłe brzegi fjordów, od południa Emil Zola rozpalał światło swoje po zaułkach życia miast i wsi, aby ztamtąd wywlec ich nędzę. (630)

[Die Literatur von „vorgestern“, wie man sie nennt, also der Naturalismus der 80-er Jahre, hatte zwei Sonnen auf seinem Horizont. Von Norden her leuchtete uns Henrik Ibsen, der mit weißem, kaltem Licht die Felsen und die gefrorenen Ufer der Fjorde beleuchtete, von Süden her wiederum warf Émile Zola sein Licht auf die Gassen des Lebens von Städten und Dörfern, um von dort her deren Not ans Tageslicht zu bringen.] 
Der Autor schreibt, dass diese beiden Schriftsteller in ihren Werken nach einer objektiven Darstellung der harten und grausamen Realität strebten, aber trotz allem bei ihrer subjektiven Wahrnehmung der Welt geblieben wären. Am Ende seines künstlerischen Schaffensweges sei Ibsen zum Mystiker geworden, während Zola seinen ästhetischen Prinzipien treu geblieben sei. Der Meinung des Verfassers nach war Zola aber immer Dichter in der wahrsten Bedeutung dieses Wortes. Dank seinem Charakter (der Autor gebraucht hier den Begriff „Temperament“) gab er seiner Umgebung Farbe, und erst in diesem Zustand präsentierte er sie seinen Lesern. Weiters schreibt der Verfasser, dass Zola sich als Fotograf verstand, der alles, was er sah, bis ins kleinste Detail darstellte. Zola habe einen starken Charakter besessen, weil seine individuelle Darstellung der Realität sehr bunt war. Somit zieht der Autor des Artikels die Schlussfolgerung: ,[... ] tylko bardzo naiwni ludzie wierzyli Zoli na słowo, że jest naturalistą" (631). [Nur sehr naive Menschen nahmen Zola seine Behauptung, er wäre ein Naturalist, auch ab.] Zum Beispiel erinnert der Verfasser an die letzte Szene aus La bête humaine, wo der französische Schriftsteller den Zug schildert, der ohne Lokomotivführer dahinfährt, in dem die Soldaten aber fröhlich singen, ohne von ihrer Tragödie zu wissen. Der Meinung des Verfassers nach reiche alleine diese Szene aus den Werken Zolas aus, um sich davon zu überzeugen, dass ein reiner Objektivismus keine solchen Figuren schaffen kann.

Der Verfasser des Artikels meint dann weiter, dass Zolas Charakter einen starken Einfluss auf seine Werke ausgeübt und sich noch während seiner Schulzeit geformt habe, als die Lehrer ihn für einen unbegabten Schüler hielten. Einmal habe Zola sogar eine Prüfung in Literatur nicht bestanden, und seine ersten literarischen Werke seien von der Kritik negativ eingestuft worden. Der Autor des Artikels ist der Meinung, dass viele Menschen an Zolas Stelle solche Schwierigkeiten nicht ausgehalten hätten, er aber seiner Umgebung standgehalten habe. In Mes haines habe Zola seine Gegner kritisiert, und dies sei auch der Anfang seines Hasses gegen alles gewesen, was er nicht akzeptierte. Der Ausruf „Ich hasse“ sei zur Quelle von Zolas schöpferischer Begeisterung geworden. Vor allem habe Zola das Elend gehasst und auf eine Weise geschildert, dass das gesamte Bild des Elends gezeigt wurde; dies laut Verfasser zur Abschreckung, damit eine derart schändliche Erscheinung im Leben der Menschen letztendlich ausgemerzt werde. Der Verfasser zitiert in diesem Kontext auch Zolas Worte über seinen Hass: ,[...] nienawidzić, to kochać, w nienawiści serce ulgę znajduje, nienawiść czyni sprawiedliwość, nienawiść nas podnosi“ (631f.). [Hassen bedeutet lieben, im Hass findet das Herz Erleichterung, der Hass bewirkt Gerechtigkeit, der Hass beflügelt uns.] Alle Werke Zolas, so der Autor des Artikels, seien voll von eigenem Hass auf die Grausamkeit und die Ungerechtigkeit des Lebens, und dieser Hass habe Zola die Kraft gegeben, sämtliche Schwierigkeiten zu überwinden, und ihn letztlich sogar von der Angst vor dem Gefängnis befreit.

Der Verfasser übt weiters Kritik an denjenigen, die über Zolas Schaffen negativ urteilten, wie etwa an Friedrich Nietzsche, der in Zolas Werken angeblich nur einen reinen Kritizismus erkennen konnte. Der Autor merkt jedoch an, dass Nietzsche sich 
auch über Victor Hugo negativ geäußert und dem großen französischen Romantiker eine übermäßig ungestüme Phantasie attestiert habe. Der Autor ist der Ansicht, dass Nietzsche damit einen extremen Standpunkt vertrete. Bezüglich der schriftstellerischen Meisterschaft von Zola behauptet der Verfasser des Artikels weiter, dass Zola selbst sich unter die Schüler von Flaubert und Balzac eingereiht habe, wobei der Verfasser dieser Klassifizierung lediglich in Bezug auf den Letztgenannten zustimmt; die ausführliche Schilderung von Tatsachen wäre nämlich das gemeinsame schriftstellerische Merkmal von Zola und Balzac, freilich habe Balzac aber kein derartiges Temperament und keine solche Vorstellungskraft wie Zola besessen. In diesem Punkt ähnle Zola Victor Hugo: Während der französische Romantiker sich aber sehr leicht von der Realität distanziere, um seine Ideale der Güte und Schönheit zu erschaffen und diese in einer irrealen Welt unterzubringen, bleibe Zola in der vom Übel übervollen Realität, um dieses zu bekämpfen. Wenn man sich kritische Anmerkungen zu Zolas Schaffen erlauben könne (so der Verfasser des Artikels weiter), dann nur wegen der wissenschaftlichen Ansichten, die der Schriftsteller manchmal zahlreich verwende (wie z. B. der Theorie der Vererbung), was wiederum die Ästhetik seiner Werke schwäche. In der Bestrebung, alle wissenschaftlichen Details darzustellen, sei Zola Voltaire ähnlich. Der Autor meint schließlich auch, dass es noch zu früh sei, das Schaffen Zolas zu bewerten und ausführlich zu analysieren.

Nach dem Artikel Émile Zola findet sich 1902 ebenfalls in Nr. 40 der „Woche“ unter der Rubrik „Prosa“ eine mit Raj dla kotów betitelte Übersetzung von Zolas Le paradis des chats und in der folgenden Nummer Nr. 41 unter dem Titel Śmierć pani Guerard eine Übersetzung von La mort de Mme. Guérard (Comment on meurt II), wobei der Name des Übersetzers nicht erwähnt wird. Man darf jedoch annehmen, dass es sich bei dem Übersetzer um den Autor des oben genannten kritischen Artikels handelte, konnte dieser doch Französisch und war mit Zolas Werken vertraut.

Auf den Seiten der ukrainischen galizischen Zeitung „Dilo“ wurde Émile Zola zum ersten Mal 1889 in Nr. 174 vorgestellt, ab dieser Nummer nämlich wurde sein ein Jahr zuvor veröffentlichter Roman Le rêve in der „Biblioteka najznamenytišych povistej“ [Bibliothek der berühmtesten Romane], redigiert von Ivan Belej, veröffentlicht. In dieser „Bibliothek“ von „Dilo“ sind insgesamt 74 Bände erschienen, hier wurden etwa Übersetzungen von Werken Balzacs, Orzeszkowas, Dickens' oder Turgenevs veröffentlicht. Von Zola selbst erschien in der „Bibliothek“ im Laufe des Jahres 1899 noch eine Übersetzung des Romans Le væu d'une morte, und ebenfalls 1899 wurde in Nr. 225 von „Dilo“ unter dem Titel Mužyka smert’ Zolas Erzählung La mort du paysan (Comment on meurt $V$ ) veröffentlicht. ${ }^{5}$ Unter der ukrainischen Übersetzung stehen als Angabe des Übersetzers lediglich die Initialen „I. S.“

Es ist zu betonen, dass in den zwei auflagenstärksten Zeitungen Galiziens, also eben im „Kurjer Lwowski“ und in „Dilo“, berühmte ukrainische und polnische

5 In Šapoval, Dilo, S. 109, fälschlicherweise als Smert' mužyka angeführt. 
Schriftsteller tätig waren, die Zolas Werke selbst übersetzten; auch hat der Naturalismus des französischen Schriftstellers ihre Werke beeinflusst. ${ }^{6}$ Das trifft auch auf Ivan Franko zu, der sowohl für die ukrainische Zeitung „Dilo“ als auch zehn Jahre lang für den polnischen „Kurjer Lwowski“ arbeitete, ehe er diesen Posten wegen der Veröffentlichung seines harschen, gegen den polnischen Nationaldichter Adam Mickiewicz gerichteten Pamphletes Ein Dichter des Verrathes in der Wiener Wochenschrift „Die Zeit“ 1897 räumen musste. ${ }^{7}$

Nach der Darstellung der Zola-Rezeption in Lemberger polnisch- und ukrainischsprachigen Tageszeitungen soll als komplementär gehaltene Ergänzung dazu mit dem „Literarisch-wissenschaftlichen Boten“ nun noch die wichtigste ukrainische Monatsschrift präsentiert werden, die zwischen 1898 und 1906 vorerst in Lemberg, danach von 1907 bis 1919 in Kiew und späterhin von 1922 bis 1932 wiederum in Lemberg erschienen ist. Die Entscheidung, die ersten fünf Jahrgänge (also von 1898 bis 1902) gerade dieses Periodikums in Hinblick auf die Rezeption Zolas durchzusehen, lässt sich dabei relativ einfach begründen: Einmal fungierte die Monatsschrift als zentrales Sammelbecken der westukrainischen Intelligenz im Verband der Donaumonarchie, die hier ihren kulturellen Bestrebungen in besonders markanter Form Ausdruck verleihen konnte. ${ }^{8}$ Eines der wesentlichen Ziele dieser Intelligenz war es, an die (als vorbildhaft empfundenen) westeuropäischen Kulturen Anschluss zu fin-

${ }^{6}$ Zur Aufnahme Zolas in Polen (ohne Rekurs auf Ivan Franko) vgl. Jan Nowakowski, Spór o Zolę w Polsce. Z dziejów pozytywistycznej recepcji naturalizmu francuskiego, Wrocław: Wydawnictwo zakładu narodowego imienia Ossolińskich 1951; zur Frage einer spezifischen Ausformung naturalistischer Ansätze in der polnischen Literatur am Ende des 19. Jahrhunderts (die sich freilich teilweise durchaus von Zola abgrenzten) vgl. weiterhin Daniel Schümann, Kunst = Natur $-\mathrm{x}$ oder Gibt es einen polnischen Naturalismus?, in: Zeitschrift für Slawistik 52 (2007) 3, S. 251-277; die beiden jeweils mit ,Émile Zola en Pologne" überschriebenen Beiträge von Janina Kulczycka-Saloni in: Les Cahiers naturalistes nr. 25 (1963), S. 111-113, bzw. nr. 32 (1966), S. 145-159, waren uns bedauerlicherweise nicht zugänglich, ebenso wenig auch V. Matviichynes bibliographische Auflistung ukrainischer Zola-Übersetzungen mit dem Titel „Émile Zola en Ukraine“ in: Les Cahiers naturalistes nr. 33 (1967), S. 68-72.

${ }^{7}$ In seinem auf Deutsch verfassten, mit „Wien, den 18. Mai 1893“ datieren umfangreichen Lebenslauf, den Franko als Doktorand der Universität Wien zusammen mit dem Ansuchen um die Zulassung zu den Rigorosen dem „Hochlöblichen K. K. Professorenkollegium der philosophischen Fakultät der Universität Wien“" vorlegte, kam der Autor dann auch auf seine Mitarbeit an beiden Lemberger Blättern zu sprechen; er schrieb in diesem Zusammenhang: ,[...] und beschäftigte mich in Lemberg, wo ich Mitarbeiter der ruthenischen Zeitschrift ,Diło“ wurde, mit dem Studium der Geschichte Galiziens sowie der galizisch-ruthenischen nationalen und litterarischen Entwickelung. [...] im Jahre 1887 wurde ich ständiger Mitarbeiter der polnischen Zeitschrift ,Kurjer Lwowski“." Zit. nach Günther Wytrzens, Ivan Franko als Student und Doktor der Wiener Universität, in: Ders., Slawische Literaturen - Österreichische Literatur(en). Hrsg. v. Fedor B. Poljakov u. Stefan Simonek, Bern u. a.: Peter Lang 2009, S. 210-226, hier S. 216.

8 Zum „Literarisch-wissenschaftlichen Boten“ der ersten Lemberger Periode von 1898 bis 1906 "vgl. Halyna Korbyč, Žurnal „Literaturno-naukovyj visnyk“ l'vivs'koho periodu (1898-1906), Kyjiv: Oberehy 1999. 
den, was sich etwa in einer beeindruckenden Anzahl an Übersetzungen aus der deutschen, französischen, englischen und italienischen Literatur und entsprechenden essayistischen Berichten in der Zeitschrift manifestierte. Ivan Franko, einer der Herausgeber des Blattes, artikulierte diese nach Europa hin ausgerichtete Tendenz der Zeitschrift in einem Vorwort zum 1903 erschienenen Registerband zu den ersten zwanzig Bänden des „Literarisch-wissenschaftlichen Boten“ in eindeutiger Weise er schreibt hier nämlich von den ursprünglichen Absichten der Herausgeber, eine Monatsschrift in Buchform nach Art einer europäischen „Revue“ herauszubringen, die dazu dienen sollte, in die ukrainische Literatur die besten geistigen und künstlerischen Strömungen der zivilisierten Welt einzuführen (III bzw. XXI). ${ }^{9}$ Zusätzlich dazu war Zolas naturalistisch ausgerichtetes Schreiben für Franko ein wichtiger Orientierungspunkt für seine eigene literarische Darstellung des Industrieproletariats in den Boryslaver Naphtagruben, wie etwa in dem Roman Boa constrictor aus dem Jahr 1878 oder dem 1881 bis 1882 in mehreren Folgen veröffentlichten, unabgeschlossen gebliebenen Romanfragment Boryslav smijet'sja [Borsylav lacht] - diese Vorbildwirkung Zolas für Franko ist in der Sekundärliteratur, wie etwa in Boris Hlynskys nach wie vor grundlegender Studie zu Franko und Zola aus dem Jahr 1979, auch nachhaltig dokumentiert. ${ }^{10}$

Die Kombination der beiden zuvor erwähnten Parameter - der generellen europäischen Ausrichtung des „Literarisch-wissenschaftlichen Boten“ und der signifikanten Relevanz Zolas für Franko - lässt für die Durchsicht der Zeitschrift entsprechende Rezeptionsbelege in Richtung Zola erwarten, und tatsächlich bestätigt sich diese Prämisse auf eindrucksvolle Weise, ja mehr noch: Die Aufnahme Zolas in den ersten Jahrgängen der Lemberger Monatsschrift verläuft - dies sei jetzt schon vorweggenommen - mit Ausnahme weniger Marginalien beinahe ausschließlich über Franko, der jeweils wechselnd als Essayist und als Übersetzer Zolas präsent ist. ${ }^{11}$

${ }^{9}$ Durch den Umstand, dass Franko das Lexem „Revue” unübersetzt und in lateinischen Lettern in seinen ukrainischen kyrillischen Text einbaut, erhält die angestrebte Integration der Zeitschrift in ein breiteres Kontinuum europäischer Kultur eine zusätzliche, auch auf der graphematischen Ebene veranschaulichte Bedeutung; daneben wird durch die Wahl eines ursprünglich aus dem Französischen stammenden Begriffs die angestrebte Ausrichtung nach Europa hin subkutan mit einer Orientierung in Richtung französischer Kultur parallel gesetzt.

10 Vgl. Boris Hlynsky, Ivan Franko et Émile Zola. Une Étude de relations littéraires, Hamburg: Helmut Buske Verlag 1979, insbes. Kapitel III. Zur Rezeption Zolas in der ukrainischen Literatur vgl. weiters die entsprechenden Passagen aus: V. I. Paščenko, H. M. Rjahuzova, Ukrajins'ko-francuz'ki literaturni vzajemyny, in: H. D. Verves (red.), Ukrajins'ka literatura v zahal'no-slov"jans'komu i svitovomu literaturnomu konteksti, t. 3, Kyjiv: Naukova dumka 1988, S. 155-184.

11 Frankos intensiv wie kontinuierlich betriebene Auseinandersetzung mit Zola beginnt klarerweise nicht erst mit der Gründung des „Literarisch-wissenschaftlichen Boten“ im Jahre 1898, sondern setzt bereits zwei Jahrzehnte zuvor ein: Schon 1876 übertrug Franko im jugendlichen Alter von zwanzig Jahren Zolas Erzählung L'indonation und veröffentlichte diese unter dem analogen ukrainischen Titel Povin' im Lemberger Almanach 
Gerade der erste Jahrgang 1898 vermag dies besonders eindrucksvoll zu belegen, bietet er doch in jedem seiner insgesamt vier Bände zentrale Rezeptionsbelege:

In Band 1 etwa betritt Zola die Zeitschrift gewissermaßen durch die Seitentüre, schreibt Franko in seiner regelmäßig erscheinenden Rubrik ,Z čužych ljiteratur،'12 [Aus den ausländischen Literaturen] hier doch nicht primär über Zola, sondern zunächst über Guy de Maupassant und etwas später dann über die deutsche Literatur der siebziger Jahre. Die erste Erwähnung findet Zola hier als Repräsentant des Naturalismus, der in der französischen Literatur der achtziger Jahre dann zu Gegenreaktionen wie Neokatholizismus und Dekadenz geführt habe; das Anwachsen von Darwinismus und Evolutionismus habe sich laut Franko auf dem Gebiet der Literatur in der gigantischen Figur Émile Zolas manifestiert und den Anstoß zu dessen Theorie des Naturalismus gegeben. Anschließend daran wird der französische Autor in $\mathrm{Zu}-$ sammenhang mit Maupassant dann in ein dichtes Netz internationaler Referenzgrößen eingebaut, das die europäische Ausrichtung des „Literarisch-wissenschaftlichen Boten" und Frankos nachdrücklich dokumentiert: Franko erwähnt hier zunächst Georg Brandes' Einschätzung von Maupassant als stilistischem Gegengewicht zum allzu farbigen Stil Zolas und kommt dann auf Zolas Rede bei der Enthüllung von Maupassants Denkmal im Pariser Park Monceau zu sprechen, bei der Zola (der von Franko hier lediglich indirekt als Autor von Germinal apostrophiert wird) seinen verstorbenen Kollegen als Gesundheit und Kraft der französischen Rasse

\footnotetext{
„Dnistrjanka“ [Das Dnjestrfräulein]; es ist dies die erste veröffentlichte ukrainische Übersetzung aus den Werken Zolas überhaupt. Ein Jahr später publizierte Franko 1877 eine knappe Charakteristik von Zolas Roman L'assommoir, der in der französischen literarischen Welt laut Franko so viel Aufsehen erregt habe wie kaum ein anderer Roman davor (Ivan Franko, Zibrannja tvoriv u 50-y tomach, t. 26, Kyjiv: Naukova dumka 1980, S. 48). 1879 verfasste Franko das Vorwort zu Ol'ha Roškevyč' in Lemberg herausgebrachter auszugsweiser Übersetzung von Zolas Roman L'assommoir, der von Franko einleitend als einer der interessantesten und wichtigsten europäischen Romane der letzten zehn Jahre gewürdigt wird (Franko, Zibrannja tvoriv, t. 26, S. 102). 1881 wiederum veröffentlichte Franko in der Zeitschrift „Svit“ [Die Welt] einen biographischen Abriss zu Zola. Franko hat sich aber nicht nur vor dem in unserem Aufsatz referierten Berichtszeitraum, sondern auch noch danach mit Zola auseinandergesetzt, wie die 1903 erschienene autobiographische Erzählung Hirčyčne zerno (Iz mojich spomyniv) [Das Senfkorn (Aus meinen Erinnerungen)] belegt, in der Franko davon berichtet, dass er im Jahre 1876 (als er ja auch seine Übersetzung von L'indonation publizierte) mit Begeisterung die ersten Romane Zolas las, die in russischen und polnischen Übersetzungen herauskamen; dies verbunden mit dem Hinweis, dass es damals angeblich noch keine deutschen Übersetzungen von Zolas Werken gab und man Zola im deutschsprachigen Raum noch gar nicht kannte (Franko, Zibrannja tvoriv, t. 21, Kyjiv 1979, S. 329). 1904 schließlich steuerte Franko zu Oleksandr Paškevyč' in Lemberg erschienener Übersetzung von Germinal ein knappes Vorwort bei, in dem er den Roman eingangs als eines der Schmuckstücke aus der Belletristik des 19. Jahrhunderts würdigte (Franko, Zibrannja tvoriv, t. 35, Kyjiv 1982, S. 334).

12 Hier und im Folgenden wird das Ukrainische in seiner im "Literaturno-naukovyj visnyk" verwendeten, von den heutigen Sprachnormen bisweilen abweichenden Form wiedergegeben. Auf eine nachträgliche Anpassung der ukrainischen Zitate an gegenwärtige Normen wurde bewusst verzichtet, um den originalen Sprachzustand zu dokumentieren.
} 
würdigte (, ,Mopassan, se zdorovlje i syla našoji rasy! · - holosyv avtor Žerminalja“ [64]). Anschließend daran zitiert Franko in extenso aus einem Artikel Max Nordaus, der gegenüber Zola wie auch gegenüber Maupassant (der hier als Paradebeispiel der „Entartung“ präsentiert wird) ausgesprochen kritisch eingestellt ist und der laut Franko den Titel Pered pamjatnykom [Vor dem Denkmal] trägt. Nordau führt hier nun seinerseits genau das von Franko gebrachte Zitat Zolas zu Maupassant als angeblicher Gesundheit und Kraft der französischen Rasse an und richtet es in polemischer Weise gegen seinen Urheber Zola, so dass dieser in Frankos Beitrag im Umweg über Nordau letztlich also gewissermaßen über ein Zitat zweiten Grades präsent ist. Das Zitat findet sich in analoger Form in dem mit Guy de Maupassant überschriebenen Kapitel aus Nordaus 1901 erschienener Essaysammlung Zeitgenössische Franzosen, in dem der (offensichtlich schon zuvor unter abweichendem Titel als Beitrag in einem Periodikum veröffentlichte) Aufsatz nochmals abgedruckt wurde; es lautet wie folgt:

Zwar ist dieser ,gute, helle und solide Kopf“ [Maupassant], wie alle Welt weiß, in einer Irrenanstalt dem fürchterlichsten Schicksal erlegen, aber wenn Zola versichert, daß der unglückliche Paralytiker die „Gesundheit und Kraft der Rasse“ ist, so wird er es ja wohl wissen $[\ldots]$. ${ }^{13}$

Nach Maupassant widmet Franko dem deutschen Impressionisten Detlev von Liliencron seine Aufmerksamkeit und reiht den Naturalismus Zolas mit dessen breit angelegten Gemälden des sozialen Kampfes unter die Anregungen, die die deutsche Literatur in den siebziger Jahren aus dem Ausland empfangen habe.

Auch die beiden darauf folgenden Verweise auf Zola verdanken sich nicht dem Autor selbst, sondern anderen Schriftstellern: In einem Bericht über Gerhart Hauptmann erwähnt Franko die literarischen Debatten über Realismus und Naturalismus bzw. über Ibsen, Zola und Tolstoj und führt Hauptmanns Drama Die Weber als Kind jenes Geistes an, der auch den Roman Germinal befruchtet habe; wenig später wird Germinal von Franko als Beispiel für ein literarisches Werk ohne individuellen Helden angeführt, in dem die Darstellung der Masse im Vordergrund stehe. In den Bereich der französischen Literatur zurück führt dann Frankos Nachruf auf Alphonse Daudet, der neben den Brüdern Goncourt, Maupassant und Zola zu einer Gruppe von Dichtern gehört habe, von der nach Daudets Ableben nun lediglich noch Zola übrig geblieben sei - dieser wird danach nun zum ersten Mal in der Zeitschrift auch in den Mittelpunkt der Darstellung gerückt, wenn Franko Zolas neuesten Roman Paris (den 1898 erschienenen Abschluss der Trilogie Trois villes) bespricht - die ersten beiden Abschnitte des Romans, die Franko bereits gelesen hat, zeigen in den

13 Max Nordau, Zeitgenössische Franzosen. Litteraturgeschichtliche Essays, Berlin: Ernst Hofmann \& Co. 1901, S. 53 f. Zu Nordau und Zola, insbesondere zum Kapitel Zola und die Zolaschulen in Nordaus Entartung vgl. Franz Norbert Mennemeier, Literatur der Jahrhundertwende I: Europäisch-deutsche Literaturtendenzen 1870-1910, Bern - Frankfurt a. M. - New York: Peter Lang 1985, S. 67 ff. 
Augen des ukrainischen Autors Zola als großen schriftstellerischen Meister und als energischen Denker (,pokazujut' nam velykoho majstra pysatelja i energičnoho myslytelja“ [131]). Danach verleiht Franko seiner Hoffnung Ausdruck, im „Literarisch-wissenschaftlichen Boten" umgehend auch Übersetzungsproben aus diesem Werk zu bringen, schildert dann in zustimmender Weise Zolas Einsatz in der Affäre Dreyfuß inklusive des offenen Briefs J'accuse und meint, dass Zola mit seinem mutigen Einsatz die Sympathien und die Bewunderung nicht nur der besten Männer Frankreichs, sondern gleichzeitig auch aller Freunde von Freiheit und Gerechtigkeit auf der ganzen zivilisierten Welt gewonnen habe (,,[Zolja] rivnočasno zdobuv sobi sympatyju i podyv ne til'ko vsjich najljipšych ljudej u Francyji, ale vsjich pryjateljiv svobody i spravedlyvosty v civiljizovanim s'vitji““ [132]).

Band 2 des ersten Jahrgangs eröffnet dann mit der Skizze Žaba [Die Kröte] die lange Reihe von Texten Zolas, die in Frankos Übersetzung im „Literarisch-wissenschaftlichen Boten" zu finden sind - der Umstand, dass diese Übersetzung direkt auf Frankos Übertragungen zweier Gedichte von Paul Verlaine, nämlich der Buß-Litanei $O$ mon Dieu vous m'avez blessé d'amour sowie des berühmten Colloque sentimental, folgt, belegt nachdrücklich die Pluralität in der ästhetischen Konzeption der Zeitschrift und nicht zuletzt auch Ivan Frankos selbst, der im Zeichen der europäischen Ausrichtung des „Literarisch-wissenschaftlichen Boten“ ganz offensichtlich sowohl den französischen Symbolismus als auch den Naturalismus in seiner Zeitschrift entsprechend repräsentiert sehen wollte. ${ }^{14}$ Sucht man nun nach der Vorlage von Frankos Übersetzung der Kröte, so stößt man auf Zolas am 28. Februar 1896 in „Le Figaro“ veröffentlichte, gegen die Instanz der Literaturkritik gerichtete Satire $\mathrm{Le}$ crapaud. Interessant ist in diesem Kontext der Umstand, dass noch im Jahr der Veröffentlichung der Satire in Nr. 83 der Wiener Wochenschrift „Die Zeit“ unter dem Titel Die Kröte eine deutsche Übersetzung des Textes (ohne Angabe des Übersetzers) erschien. ${ }^{15}$ Ein Vergleich dieser Übersetzung mit jener Frankos zeigt recht bald, dass Franko ungeachtet der Möglichkeit einer Übertragung des Textes über eine deutsche Zwischenstufe Zola dennoch direkt aus dem Französischen übersetzt haben muss. So schreibt Zola im vorletzten Absatz seines Textes etwa: „Tombe, tombe donc toujours chez moi, bienfaisante pluie de crapauds!“ oder „Et, le jour où mon crapaud me manquera, c'est que ma fin sera prochaine et que ma dernière bonne page sera écrite.“16 In der Übersetzung der „Zeit“" dagegen fehlen die kursiv gesetzten Passagen, es heißt hier: „So ströme denn, ströme nur zu, wohlthuender Re-

${ }^{14} \mathrm{Zu}$ Frankos Übersetzungen von Gedichten Verlaines vgl. Stefan Simonek, Ivan Franko und die „Moloda Muza“. Motive in der westukrainischen Lyrik der Moderne, Köln Weimar - Wien: Böhlau 1997, S. 157-160.

15 Zur Rezeption Zolas in der „Zeit“" vgl. Karl Zieger, Die Aufnahme der Werke von Emile Zola durch die österreichische Literaturkritik der Jahrhundertwende, Bern - Frankfurt a. M. - New York: Peter Lang 1986, S. 181-191.

16 Émile Zola, Nouvelle campagne 1896, Paris: Eugène Fasquelle 1897, S. 80 f. Die Kursivsetzungen hier und in den folgenden Zitaten sämtlich von S. Simonek. 
gen der Kröten!“ bzw. „Und fehlt mir eines Tages meine Kröte, dann ist meine letzte gute Seite geschrieben." ${ }^{\text {17 }}$ In Frankos Übersetzung wiederum sind die hier ausgesparten Passagen des französischen Originals sehr wohl übertragen worden, was eben auf das Original als Vorlage der Übersetzung hindeutet, vgl.: „Pady-ž raz-u-raz na mene, dobrodjijna žabjača zlyvo!“ bzw. „I koly odnoho dnja ne stane menji toji žaby, to se bude znak, ščo blyz'kyj mij konec', ščo napysana ostatnja moja harna storinka“" $(361) .{ }^{18}$

Auch wenn Franko Zolas Satire gegen den Literaturbetrieb nun ganz offensichtlich direkt aus dem Französischen übertragen hat, so könnte er dazu doch durchaus von der Übersetzung in der Wiener "Zeit" angeregt worden sein, hat Franko für dieses Blatt zwischen 1894 und 1904 insgesamt doch rund dreißig Beiträge meist zu ökonomischen, politischen und sozialen Fragen Galiziens veröffentlicht, darunter 1896 in Nr. 72 etwa den Aufsatz Die erste Session des galizischen Landtags oder in Nr. 88 (und damit nur fünf Nummern nach Zolas Die Kröte in Nr. 83) die Satire Das Recht des Schweins. ${ }^{19}$ Die Vorbildwirkung, die der Wiener ,Zeit“ für den vier Jahre später gegründeten Lemberger „Literarisch-wissenschaftlichen Boten“ zugekommen ist, findet sich in der mehrfachen Übernahme von Beiträgen aus der „Zeit“ durch den „Boten“ auch eindrücklich dokumentiert, so dass Franko mit seiner vielleicht durch die „Zeit“ inspirierten Übertragung von Zolas Le crapaud indirekt auch an der Aufnahme Zolas im kulturellen Zentrum Wien partizipierte. ${ }^{20}$

Ebenfalls in Band 2/1898 des „Literarisch-wissenschaftlichen Boten“ vergleicht Franko in seiner Aufsatzfolge zu den ausländischen Literaturen dann späterhin Zola

17 Emile Zola, Die Kröte, in: Die Zeit Nr. 83, Wien, Samstag, 2. Mai 1896, S. 74.

18 Auch der von Hlynsky, Ivan Franko et Émile Zola, S. 211, angeführte Übersetzungsfehler Frankos, der Zolas Wendung „l'article d'un sectaire, détraqué de la politique ou de la foi“ in seinem ukrainischen Text sinngemäß als „Artikel eines Sektierers, der alle Verbindungen zur Politik und zum Glauben hin abgebrochen hat" wiedergibt, findet in der deutschen Übersetzung der „Zeit" keine Vorgabe. Hier heißt es nämlich gänzlich anders lautend: „Dann gibt es den wahnwitzigen Artikel. Ich meine damit den Artikel eines Sectierers, eines politisch oder religiösen Fanatikers.“ Zola, Die Kröte, S. 74.

19 Ein Großteil dieser Beiträge liegt gesammelt vor in: Ivan Franko, Beiträge zur Geschichte und Kultur der Ukraine. Ausgewählte deutsche Schriften des revolutionären Demokraten 1882-1915. Hrsg. v. Eduard Winter u. Paul Kirchner, Berlin-Ost: Akademie-Verlag 1963. In seinen deutschsprachigen Beiträgen äußerte sich Franko mindestens zweimal zu Zola, einmal in seinem bereits zitierten, 1893 an der Universität Wien eingereichten Lebenslauf, wo Franko eine eigene, auf Polnisch verfasste Arbeit erwähnt, in der er Zolas La terre mit der Skizzensammlung Vlast' zemli [Die Macht der Scholle] des russischen Autors Gleb Uspenskij vergleicht, dem Franko in Nr. 406 der „Zeit“ vom 12. Juli 1902 fast ein Jahrzehnt später dann auch einen Nachruf gewidmet hat, vgl. Wytrzens, Ivan Franko als Student und Doktor, S. 219. In dem autobiographischen Beitrag Wie ich dazu kam?, der am 17. März 1903 in Nr. 167 der ,Zeit" veröffentlicht wurde, vermerkte Franko wiederum ein rundes Dezennium nach seinem Lebenslauf aus dem Jahre 1893: „Ich las Dickens, Tolstoj und Zola [...]." Franko, Beiträge, S. 60.

20 Vgl. zu dieser Frage Norbert Bachleitner, La traduction de Zola à Vienne. Marché littéraire, censure et goût bourgeois, in: Les Cahiers naturalistes nr. 81 (2007), 169-179. 
erneut mit Daudet und lobt dessen sarkastischen Humor, der bei Zola vollständig fehle. Für Franko ist Daudet der größere Pessimist als Zola, dafür zeichnen sich seine Skizzen aber durch Phantasie und Gefühl aus, so dass sie laut Franko wie Paradiesgärten aussehen, wenn man sie an Zolas graue und düstere kolossale Panoramen halte.

Die von Franko in Band 1 in Aussicht gestellte Textprobe aus dem Roman Paris bietet dann schließlich Band 3 der Zeitschrift, wo sich unter dem Titel Zločynec' Sal'vá [Der Übeltäter Salvat] vier mit den von Franko eigenmächtig gewählten, im französischen Original nicht vorhandenen Titeln Hnjizdo [Das Nest], Zločyn [Die Untat], Pohonja [Die Verfolgungsjagd] und Sud [Das Gericht] überschriebene Ausschnitte aus dem Roman finden. In einer als Fußnote gesetzten Anmerkung positioniert Franko die einzelnen Abschnitte innerhalb der Romanhandlung von Paris und weist dann noch eigens darauf hin, dass es sich bei den Textproben um besonders gelungene, teilweise gekürzte Abschnitte aus dem neuen Werk des großen französischen Naturalisten handle, das hier freilich de facto in Form von mehreren Einzelerzählungen, nicht aber in der epischen Großform des Romans präsentiert wird. Ein fünfter und letzter, mit Kara [Die Strafe] betitelter Auszug aus dem Roman $^{21}$ ist dann bereits im Folgeband enthalten, der mit einer groß angelegten, mehr als dreißig Seiten starken Würdigung Zolas durch Franko (wiederum unter der Rubrik „Aus fremden Literaturen“) sicherlich das Herzstück der Zola-Rezeption im „Literarischwissenschaftlichen Boten" mindestens in seinen ersten Jahrgängen darstellt. Franko bietet hier einen Abriss von Zolas Biographie und eine (jeweils einen längeren oder kürzeren Absatz umfassende) Charakteristik seiner wichtigsten Werke, wobei der französische Autor von Franko als der heute zweifellos klangvollste Name in der Republik der Literatur weltweit gewürdigt und folgende Texte Zolas im Einzelnen präsentiert werden: La fortune des Rougons, La curée, Le ventre de Paris, La conquête de Plassans, La faute de l'abbé Mouret, Son Excellence Eugéne Rougon, L'assommoir, Une page d'amour, Nana, Pot-bouille, Au bonheur des dames, La joie de vivre, Germinal, L'œuvre, La terre, La bête humaine, L'argent und La débâcle. Außerdem beinhaltet der Beitrag Frankos noch ein Porträt Zolas sowie ein umfangreicheres Zitat aus Benno Diederichs 1898 in Leizig erschienener Studie zu Zola. Frankos eigene Position zwischen Naturalismus und Symbolismus geht übrigens eindeutig aus der Tatsache hervor, dass der gesamte Beitrag von seinem Titel Emil' Zolja, jeho žytje i pysanja - Stefan Malljarme [Émile Zola, sein Leben und Werk Stéphane Mallarmé] her eigentlich zu gleichen Teilen zwei französischen Schriftstellern - nämlich Zola und Mallarmé - gewidmet sein sollte, sich Mallarmé als Repräsentant des Symbolismus aber mit dem letzten, etwas umfangreicher gehaltenen

${ }^{21}$ Zur (auf die Seite genauen) Zuordnung der fünf von Franko ausgewählten Abschnitte zur Ausgabe von Paris durch Maurice Le Blond vgl. Hlynsky, Ivan Franko et Émile Zola, S. 327. 
Absatz des Textes begnügen muss; dieser stellt einen Nachruf auf den am 19. September 1898 verstorbenen französischen Dichter dar, den Franko seinen eigenen Worten zufolge lediglich aus der Pflicht des Chronisten heraus verfasst hat und in dem er Mallarmé die angebliche Unverständlichkeit seiner Lyrik für ein breiteres Publikum zum Vorwurf macht.

Die herausragende Stellung, die Zola für Frankos ästhetische Programmatik beanspruchen konnte, geht auch aus dem zweiten Band im zweiten Jahrgang der Zeitschrift hervor, in dem ein Teil von Frankos wichtigem Aufsatz zu den Geheimnissen des dichterischen Schaffens (so der Titel) abgedruckt ist; in jenem Kapitel dieser Abhandlung, das der Rolle der Sinne im poetischen Schaffen gewidmet ist, greift Franko zur Veranschaulichung des Geruchs auf die Käsesorten in Le ventre de Paris sowie auf die Blumendüfte in La faute de l'abbé Mouret zurück. Eine scheinbar marginale Notiz in der Zeitschriftenschau des Bandes belegt dann die Zirkulation von Übersetzungen innerhalb der Zeitschriftenlandschaft Galiziens und der Bukowina, verweist sie doch auf den Nachdruck der Übersetzung von Zolas Le crapaud durch Franko aus Band 2/1898 in den Nummern 102 und 103 der Czernowitzer Tageszeitung „Bukovyna“.

Ein weiterer Beitrag aus ebendiesem Band 6/1899 vermag das bisher gewonnene Bild zu erweitern und zeigt, dass die Auseinandersetzung mit Zola im „Literarisch-wissenschaftlichen Boten" mindestens abschnittsweise auch über die Aktivitäten Ivan Frankos hinausgehen konnte. Eine einigermaßen unerwartete Erwähnung Zolas, die belegt, wie eng die Lemberger Monatsschrift mit literarischen und publizistischen Institutionen in den übrigen Regionen der Donaumonarchie verbunden gewesen ist, bietet die Übersetzung einer Übersichtsdarstellung zur zeitgenössischen serbischen, slowenischen und slowakischen Literatur aus dem ersten Jahrgang (1899) des in Prag herausgebrachten tschechischen „Slovanský Přehled“ [Die slawische Revue], in dem der bekannte slowenische Kritiker Fran Vidic über die zeitgenössische slowenische Literatur berichtet; dabei kommt Vidic auch auf den slowenischen Naturalisten Fran Govekar zu sprechen und skizziert dessen Position gegenüber Zola. ${ }^{22}$ In-

${ }^{22}$ Zur Aufnahme Zolas durch Govekar speziell im Roman $V$ krvi [Im Blut] vgl. Evald Koren, Govekar, Zola in „V krvi“, in: Slavistična revija 21 (1973) 3, S. 281-319; Janko Kos, Primerjalna zgodovina slovenske literature, Ljubljana: Znanstveni institut Filozofske fakultete / Partizanska knjiga 1987, S. 158; Tone Smolej, Govekarjeva afirmacija Émila Zolaja, in: Slavistična revija 52 (2004) 3, S. 295-311. Über den Prager „Slovanský Přehled“ kann ausgehend von Fran Vidic noch eine weitere Verbindungslinie nach Wien gezogen werden, die nun aber nicht vorrangig in das slowenische, sondern vielmehr in das kroatische kulturelle Milieu der Hauptstadt hineinzielt: 1898 erschien in Wien der erste Band der kurzlebigen kroatischen Zeitschrift „Mladost“ [Die Jugend], die im Zeichen ihrer gesamtsüdslawischen Bestrebungen neben kroatischen auch serbische und slowenische Texte abdruckte, darunter auch den Beitrag $O$ novejši slovenski literaturi [Über die neueste slowenische Literatur] von Fran Vidic, in dem sich ebenfalls der Vergleich zwischen Zola und Govekar erwähnt findet. Über die „Mladost“ wiederum ergibt sich ein direkter Kontakt zu Zola, wurde dieser von der Zeitschrift doch zur Mitarbeit eingeladen. Am 1. De- 
teressant ist darüber hinaus die starke Präsenz Frankos in der „Slawischen Revue“, die sich etwa in seiner Darstellung der ukrainischen Literatur des Jahres 1898 manifestiert, in der Franko auch auf die Gründung des „Literaturno-naukovyj visnyk“ zu sprechen kommt und dazu bemerkt, dass die neue Zeitschrift Übersetzungen aus fremden zeitgenössischen Literaturen veröffentliche, und dies ohne Rücksicht auf irgendeine Schule oder irgendwelche Formeln und einzig unter Beachtung des literarischen Wertes der ausgewählten Texte $(,[\ldots]$ neváže se na žádnou školu, žádné formulky, přihlížeje toliko k literární ceně spisův“ [388 f.]). ${ }^{23}$ Die offene, keinem dezidierten ästhetischen oder weltanschaulichen Programm verpflichtete Blattlinie des „Boten“, vor deren Hintergrund die Berichterstattung zu Émile Zola insgesamt zu sehen ist, wird von Franko also auch nach außen hin in einer Darstellung für ein nicht-ukrainisches (in diesem Falle tschechisches) Publikum besonders betont.

In eine ähnliche, wie im Falle von Fran Vidic über Franko selbst hinausgehende Richtung verweist in Band acht des „Literarisch-wissenschaftlichen Boten“ dann auch ein Beitrag zum literarischen Vermächtnis Daudets aus der Feder des bekannten ukrainischen Historikers und Literaturwissenschaftlers Mychajlo Hruševs'kyj, in dem Zola dreimal zu Daudet in Relation gebracht wird - zuerst über ökonomische Parameter, wenn die Auflagenzahlen von Daudets 1884 veröffentlichtem Roman Sapho an jenen von Zolas erfolgreichsten Romanen gemessen werden, danach in der Ablehnung durch das kulturpolitische Establishment - Hruševs'kyj erwähnt, dass weder Daudet, noch Flaubert oder Zola zu Mitgliedern der „Académie française“ gewählt wurden - und schließlich über die Texte selbst: Hruševs'kyj stellt die drei erwähnten französischen Autoren in Relation zueinander und meint, dass Daudet zwar nicht die Tiefe und die Vielseitigkeit von Flaubert und Zola erreicht habe, die beiden dafür aber in der leichten Grazie seines Erzählens überrage. ${ }^{24}$

zember 1897 reagierte Zola in einem an Guido Jeny, einen der Redakteure der „Mladost“, gerichteten Brief aus Paris auf dieses Angebot wie folgt: „Je vous donne volontiers l'autorisation de traduire pour votre revue en langue croate quelques pages détachées de mon œuvres, et je souhaite bon succés à votre enterprise“. Zitiert nach: Stanislav Marijanović, Fin de siècle hrvatske Moderne (Generacije „mladih“ i časopis „Mladost“), Osijek: Štampa 1990, S. 203 (freundlicher Hinweis von Andreas Leben, Wien).

23 Weiters beinhaltet der erste Jahrgang des „Slovanský přehled“ noch eine diachron gehaltene Übersichtsdarstellung Frankos zur ukrainischen Literatur mit dem Titel Literatura ukrajinsko-ruská (maloruská) [Die ukrainisch-russische (kleinrussische) Literatur], ein Porträt des Autors und einen Bericht über eine Feier anlässlich von Frankos 25-jährigem Jubiläum als Schriftsteller, die in Lemberg veranstaltet wurde.

${ }^{24} \mathrm{Zu}$ der in den eingesehenen Jahrgängen des "Literarisch-wissenschaftlichen Boten“ durchgängig zu beobachtenden Kombination der Namen Zola, Daudet und Maupassant vgl. auch folgende Beobachtung von Norbert Bachleitner: „Dans la monarchie austrohongroise, on peut même constater un assez vif intérêt pour le naturalisme. Daudet, Maupassant et Zola y trouvent une tribune permanente dans des revues et des journaux, y compris la ,Nouvelle Presse libre“, le journal le plus célèbre de la monarchie." Bachleitner, La traduction de Zola, S. 171. - Es steht zu vermuten, dass Franko in einem dieser Blätter auch die zuvor zitierte Invektive Max Nordaus in Richtung Maupassant gefunden hat, war Nordau u. a. doch etwa auch Korrespondent der „Neuen Freien Presse“. 
Wir kommen damit zum dritten Jahrgang des „Literarisch-wissenschaftlichen Boten“, der systematisch auf die Rezeption Zolas hin durchgesehen wurde - der erste Band im Jahr 1900 (insgesamt Band neun der Zeitschrift) steht diesbezüglich ganz eindeutig im Zeichen der Auseinandersetzung mit Zolas Roman Fécondité, dem 1899 erschienenen ersten Teil der Tetralogie Les quatre évangiles. Franko bringt vorerst eine von ihm selbst übertragene Skizze aus dem Roman mit dem Titel Miščanyn i seljanyn [Städter und Landmann]; den Angaben Boris Hlynskys zufolge umfasst der von Franko ausgewählte Ausschnitt die Seiten 217 bis 228 in der Ausgabe von Fécondité durch Maurice Le Blond und beinhaltet im Wesentlichen ein Gespräch zwischen Mathieu Froment und dem Müller Lepailleur über die Notwendigkeit, die Erde zu kultivieren. ${ }^{25}$ Ungeachtet dieser raschen Präsentation einer ukrainischen Übersetzungsprobe aus Zolas neuem Roman übt Franko in seiner Rubrik „Aus fremden Literaturen“ anders als in den früheren Jahrgängen des „Literarischwissenschaftlichen Boten", wo Zola stets rein zustimmend bis begeistert besprochen wurde, nun erstmals auch offene Kritik an Zolas schriftstellerischen Fähigkeiten. Konkret stößt sich Franko an der Thesenhaftigkeit und Schablonenhaftigkeit des neuen Romans, der in seinen Augen eigentlich keinen Roman, sondern ein publizistisches Traktat, einen kolossalen Vortrag mit Beispielen zu einer einzigen wichtigen und komplexen Frage des sozialen Lebens darstellt. In den Augen Frankos geraten hier einander Zola als Romancier und Zola als Soziologe bei jedem Schritt in die Quere - der Soziologe sammle für den Romancier das Material und gebe diesem die Linie vor, der Romancier wiederum habe die schwierige Aufgabe, diese a priori gewählte Konstruktion dann auch entsprechend mit Leben zu erfüllen. Bei aller Weite des Blicks und bei aller Lebendigkeit der Phantasie repräsentiert Zola für Franko dennoch keinen wirklich tiefen Denker, stattdessen aber einen reichlich naiven Soziologen, und es ist für Franko daher auch nicht verwunderlich, wenn der wissenschaftliche Ertrag von Zolas Romanen nicht mit den Verlusten mithalten könne, die der Künstler erleiden muss. Klar ersichtlich wird diese Argumentationslinie auch aus Frankos Bemerkung „Artyst ne mih uporaty sja z zadačeju, pokladenoju jomu socyol'ogom“ (56). [Der Künstler vermochte nicht mit der ihm vom Soziologen gestellten Aufgabe fertig zu werden.] Band 10/1900 bietet in Richtung Émile Zola le-

${ }^{25}$ Hlynsky, Ivan Franko et Émile Zola, S. 327 bzw. 204; im vierten Kapitel seiner Studie, das Franko als Übersetzer Zolas gewidmet ist, kommt Hlynsky abschließend auch kurz auf den erwähnten Ausschnitt aus Fécondité zu sprechen und vermerkt hier Frankos Wiedergabe der Wendung „c'est fini“" mit dem deutschen, in Galizien damals gebräuchlichen „kaput“ (S. 224), was Frankos (in diesem Fall indirekt präsente) Mehrsprachigkeit und sein ständiges Hin- und Herwechseln zwischen den in Galizien gebräuchlichen Sprachen Ukrainisch, Polnisch und Deutsch belegt. Vgl. zu dieser Problematik auch Juliane Besters-Dilger: Deutsch-galizische lexikalische Sprachbeziehungen (mit besonderer Berücksichtigung der Austriazismen), in: Ethnoslavica. Festschrift für Gerhard Neweklowsky zum 65. Geburtstag. Hrsg. v. Johannes Reinhart u. Tilmann Reuther, Wien: Gesellschaft zur Förderung slawistischer Studien 2006, S. 29-42. 
diglich eine Notiz in der Zeitschriftenschau des Bandes, in der auf die bereits erwähnte Übersetzung von La mort du paysan unter dem analogen Titel Mužyka smert' in Nr. 225 von „Dilo“ hingewiesen wird.

Ganz offensichtlich hat das oben artikulierte, deutliche Missfallen an Zolas Roman Fécondité Franko dazu veranlasst, von einer weiteren intensiven Auseinandersetzung mit dem französischen Autor Abstand zu nehmen, wie die Jahrgänge 1901 und 1902 des „Literarisch-wissenschaftlichen Boten“ mit Nachdruck belegen: In ihnen findet sich nämlich in signifikantem Kontrast zu den ersten drei Jahrgängen der Zeitschrift kaum mehr ein relevanter Rezeptionsbeleg in Richtung Zola. Auf den Seiten des Lemberger Blattes treten gegenüber dem französischen Naturalisten nun die gesamteuropäische Moderne, insbesondere August Strindberg, aber auch die wichtigsten Stimmen der eigenen, west- wie ostukrainischen Moderne, wie etwa Petro Karmans'kyj oder Lesja Ukrajinka, deutlich in der Vordergrund des Interesses, Franko selbst veröffentlicht Übersetzungen entweder aus Victor Hugo oder aber aus Georges Rodenbach ${ }^{26}$ und stellt an das Ende des siebenten Abschnitts seiner Abhandlung $Z$ ostatnjich desjatyljit' XIX $v$. [Aus den letzten Jahrzehnten des 19. Jhs.] eine umfangreiche Textprobe von Henrik Ibsen. Dass Franko ungeachtet dieser offensichtlichen Neuausrichtung der eigenen ästhetischen Parameter in Richtung Moderne auf der anderen Seite dennoch weiterhin die zentrale Vermittlungsinstanz für Émile Zola geblieben ist, belegt der Umstand, dass die einzigen beiden bei der Durchsicht der entsprechenden vier Bände des Jahres 1901 und der ersten drei Bände des Jahres 1902 registrierten Rezeptionsbelege dann doch wiederum mit Franko verbunden sind: In Band 14/1901 findet sich unter der Rubrik „Novi knyžky“ [Neue Bücher] eine Notiz zu Frankos unter dem entsprechenden Titel Napad na mlyn 1901 in Lemberg erschienener Übersetzung von Zolas L'attaque du moulin, in Band 18/1902 unter derselben Rubrik eine analoge Notiz zu Frankos Übersetzung von Zolas L'indonation, die im Jahr 1902 in Lemberg zwar wiederum unter dem Titel Povin', anders als noch 1876 diesmal aber als Einzelveröffentlichung erschienen ist.

In Band 20/1902 schließlich wird das Ableben Zolas am 29. September dieses Jahres unter den Nekrologen vermerkt. In einem lediglich mit „V.“ unterzeichneten und daher wahrscheinlich nicht von Franko stammenden Nachruf wird Zola eingangs zwar als einer der wichtigsten Schriftsteller der Welt gelobt, danach finden sich unter Verweis auf einen bereits veröffentlichten größeren Aufsatz zu Zola, mit dem wohl Frankos Würdigung aus dem Jahr 1898 gemeint sein dürfte, aber lediglich ein biographischer Abriss und eine knappe Aufzählung von Zolas wichtigsten Werken; insbesondere wird hier der Zyklus Les Rougon-Macquart hervorgehoben, dessen einzelne Teile laut „V.“ zwar nicht alle gleich gelungen sind, von denen einige

${ }^{26}$ Vgl. dazu Jarema Kravec', Bel'hijs'kyj pys'mennyk Žorž Rodenbach v interpretaciji Ivana Franka, in: Ivan Franko - pys'mennyk, myslytel', hromadjanyn. Materialy Mižnarodnoji naukovoji konferenciji (L'viv, 25-27 veresnja 1996 r.), L'viv: Svit 1998, S. 820824. 
aber Zola in die erste Reihe nicht nur der französischen Autoren, sondern der Schriftsteller der ganzen Welt rücken. An einzelnen Werken Zolas werden dann folgende Arbeiten aufgelistet: La fortune des Rougon, La curée, Le ventre de Paris, La conquête de Plassans, La faute de l'abbé Mouret, Son Excellence Eugén Rougon, L'assommoir, Une page d'amour, Nana, Pot-bouille, Au bonheur des dames, La joie de vivre, Germinal, L'œuvre, La terre, Le rêve, La bête humaine, L'argent, La debâcle und schließlich Le docteur Pascal. Interessant scheint auch der darauf folgende Hinweis auf bereits ins Ukrainische übertragene Werke Zolas. Die abschließende Ankündigung des Nekrologs, Ausschnitte von Zolas letztem, unvollendet gebliebenen Roman La justice noch im selben Band der Zeitschrift zu veröffentlichen, fand in einem zwanzigseitigen Auszug aus dem Roman in der Übersetzung von Franko - dessen zentrale Vermittlerrolle in Bezug auf Zola damit ein weiteres Mal bestätigt wird - ihre Realisierung. ${ }^{27}$

Insgesamt dokumentieren die hier aufgelisteten Rezeptionsbelege zu Émile Zola aus den Lemberger Periodika im zeitlichen Umkreis der Jahrhundertwende die intensive Aufnahme des französischen Schriftstellers in der polnischen und ukrainischen Publizistik und Literatur in Galizien um 1900; in dieser Hinsicht stand Zola für ein ästhetisches Modell gesellschaftsanalytisch-naturalistischen Schreibens, das sich im Zeitraum zwischen 1875 und 1900 besonders für Ivan Franko als relevant auch für die Abfassung eigener belletristischer Arbeiten wie etwa Boa constrictor erwies, bis sich Franko schließlich - begleitet von seiner Kritik an Zolas künstlerischen Fähigkeiten, wie sie am Beispiel der negativen Beurteilung von Fécondité ersichtlich wird - anderen, stärker der europäischen Moderne verpflichteten literarischen Mustern zuwandte. ${ }^{28}$ Darüber hinaus verweist die Beschäftigung Frankos mit Zola, wie sie insbesondere in den ersten drei Jahrgängen des „Literarisch-wissenschaftlichen Boten" nachdrücklich dokumentiert ist, auf die engen Verbindungen zwischen dem kulturellen und publizistischen Leben in Lemberg auf der einen und jenem in Wien auf der anderen Seite, mithin also zwischen Zentrum und Peripherie der Donaumonarchie: Ausgehend von einem dicht geknüpften Netz an persönlichen wie institutionellen Verbindungen (wie etwa Frankos Studium in Wien und seine auch nach der Promotion im Jahre 1893 weitergeführten Kontakte zu seinem akade-

${ }^{27}$ Von den insgesamt acht Bänden der Jahrgänge 1903 und 1904 des „Literarisch-wissenschaftlichen Boten“ wurden jeweils noch die Inhaltsverzeichnisse durchgesehen; hier findet sich weder eine Übersetzung aus dem Werk Zolas noch ein essayistischer Beitrag zu ihm, was Frankos sich ja bereits in den Bänden der Jahrgänge 1901 und 1902 ankündigendes, allmählich erlahmendes Interesse an dem französischen Autor belegt.

28 Tamara Hundorova gliedert in ihrer grundlegenden Monographie zu Ivan Franko das Schaffen des Autors chronologisch in Jahrzehnte, denen dann jeweils ein zentraler geistesgeschichtlicher Begriff zugeordnet wird. Für die 1880-er Jahre ist dies eben der Naturalismus (mit Hinweis auf Frankos produktive Auseinandersetzung mit Zolas Roman L'assommoir in seinen das Boryslaver Naphtarevier thematisierenden Werken); für die 1890-er Jahre wäre dies laut Hundorova dann die Psychoanalyse, vgl. Tamara Hundorova, Franko - ne kamenjar, Melbourne: Monash University, Slavic Section 1996, S. 27. 
mischen Lehrer Vatroslav Jagić ${ }^{29}$ sowie Frankos anhaltende publizistische Tätigkeit für Wiener Blätter wie die „Zeit““ oder die „Arbeiter-Zeitung ${ }^{630}$ ) führte die Rezeption Zolas im urbanen multinationalen und mehrsprachigen Milieu Lembergs zu einem nicht unwesentlichen Teil auch über die Reichshauptstadt Wien, über deren publizistische und literarische Kanäle die Aufnahme Zolas in der Hauptstadt Galiziens mindestens teilweise präfiguriert wurde - was auf der anderen Seite freilich direkte, unmittelbar in Frankreich ihren Ausgang nehmende Rezeptionsverläufe keinesfalls ausschloss.

Neben Frankos erwähntem Zitat aus einem Artikel Max Nordaus zu Guy de Maupassant, der die zuvor angesprochene deutschsprachige Zwischenstufe in der Lemberger Zola-Rezeption eindeutig belegt, vermag die im Vorangegangenen ebenfalls thematisierte Übersetzung von Zolas gegen den Literaturbetrieb gerichteter Satire Le crapaud durch Ivan Franko gerade die Multipolarität der Aufnahme Zolas in Galizien zu dokumentieren: Der Impuls, gerade diesen Text Zolas ins Ukrainische zu übertragen und 1898 dann in Band zwei des „Literaturno-naukovyj visnyk“ zu veröffentlichen, konnte für Franko wohl von der deutschen Übersetzung von Zolas Satire herrühren, die Anfang Mai 1896 unter dem Titel Die Kröte in der Wiener „Zeit“ publiziert wurde; für die simultane Präsenz direkter Rezeptionswege spricht hingegen der Umstand, dass Zola den Text ganz offensichtlich nicht über diese deutschsprachige Zwischenstufe, sondern unmittelbar von der französischen Vorlage weg übertragen hat. Neben diese direkte Achse zwischen Paris, Wien und Lemberg und die Bipolarität von „Zeit“ und „Literarisch-wissenschaftlichem Boten“ tritt daneben aber noch das Konvergenz- wie Konkurrenzverhältnis zwischen polnischen und ukrainischen Medien in Lemberg selbst, von dem am Beginn dieses Beitrags in der Darstellung des polnisches Blattes „Kurjer Lwowski“ und der ukrainischen Zeitung „Dilo“ in Bezug auf Zola die Rede war. Ergänzt wird dieses dichte Gewebe von Vermittlungslinien dann schließlich im Zeichen indirekter, gebrochener Rezeptionsverläufe zusätzlich noch durch die Linie Laibach - Wien - Prag - Lemberg, die von Fran Vidic' Erläuterungen zur zeitgenössischen slowenischen Literatur in der Wiener „Mladost“ über den Prager „Slovanský Přehled“ wiederum nach Lemberg verläuft und sich dort in der ukrainischen Übersetzung des betreffenden Artikels im „Literarisch-wissenschaftlichen Boten“ manifestiert findet, wobei Émile Zola in Zusammenhang mit dem slowenischen Naturalisten Fran Govekar genannt wird. Über diese gleichzeitige Präsenz verschiedener externer Orientierungspunkte erweist sich die Aufnahme Zolas in den drei hier eingesehenen Lemberger Periodika als ein weiterer Beleg für die ungebrochene Relevanz des von Zoran Konstantinović 1979 skiz-

${ }^{29}$ Vgl. auch die abgedruckte Korrespondenz zwischen Franko und Jagić bei Marian Jakóbiec, Iwan Franko i Vatrosłav Jagić, in: Slavia Orientalis VIII (1959) 2-3, S. 67-90.

30 Vgl. dazu Stefan Simonek, Ivan Franko in der Wiener ,Arbeiter-Zeitung“ (1889-1901) (Addenda et corrigenda), in: Wiener Slavistisches Jahrbuch 52 (2006), S. 201-212; ders., Ivan Franko als Mitarbeiter der ,Zeit“" (im Druck). 
zierten „europäischen Zwischenfeldes“ mit seinen heterogenen internationalen kulturellen Orientierungspunkten ${ }^{31}$ sowie für die Funktionalisierbarkeit des theoretischen Ansatzes der Pluralität, wie ihn Moritz Csáky in einem zentralen Aufsatz des Jahres 1996 für die kulturelle Konstellation Zentraleuropas skizziert hat: Die von Csáky in diesem Kontext vermerkte ,ständige Präsenz des Differenten“32 artikuliert sich ja gerade auch in den zwischen Paris, Wien, Laibach, Prag und Lemberg verlaufenden, über mehrere Sprachen führenden Rezeptionswegen in Sachen Émile Zola auf nachdrückliche Weise. Wenn all diese heterogenen und vielfältigen Rezeptionslinien letztlich bei Ivan Franko zusammenlaufen und von diesem gebündelt, ja beinahe monopolisiert werden (betrachtet man die geringe Anzahl an Rezeptionsbelegen zu Zola im „Literarisch-wissenschaftlichen Boten“, die nicht mit Franko verbunden sind), so lässt sich dies im Übrigen durchaus als eine weitere Ausformung von Pluralität im Sinne einer ständigen Präsenz des Differenten verstehen.

A b st ra ct: The reception of Emile Zola in Lemberg's periodicals around 1900. The essay offers a detailed bibliographical overview on the reception of Émile Zola's works in selected periodicals edited in Lviv around 1900. In the Polish daily "Kurjer Lwowski" we regularly can find translations of Zola's latest stories and novels as well as essays on the French novelist and his work. This also is true for the Ukrainian daily "Dilo" and the prestigious Ukrainian journal "Literaturno-naukovyi visnyk". In the journal, Zola is presented almost exclusively by Ivan Franko who not only translated Zola's stories and selected chapters of his novels (as, for example, Paris) into Ukrainian, but also presented Zola and several aspects of his writing in his own essays. Up to the turn of the century, Franko in all his critiques always agreed with Zola, but in 1900, for the first time he criticised him for his lack of inspiration in Fecondité, the French writer's latest novel. This also put an end to Franko's earlier initiatives to present Zola in his journal.

K e y w or d s : Émile Zola, Ivan Franko, Lviv periodicals

Stefan Simonek

Institut für Slawistik der Universität Wien

Universitätscampus AAKH, Hof 3

Spitalgasse 2, 1090 Wien, Österreich

stefan.simonek@univie.ac.at
Natalia Tsikra

Am Kanal 147/1/34

1110 Wien, Österreich natalia@tsikra.com

31 Zoran Konstantinović, Das europäische Zwischenfeld. Von einer Schwerpunktbildung der österreichischen Komparatistik, in: Sprachkunst X (1979), S. 69-78.

32 Moritz Csáky, Pluralität. Bemerkungen zum ,dichten System“ der zentraleuropäischen Region, in: Neohelicon XXIII (1996) 1, S. 9-30, hier S. 10. 